\title{
Performance Optimisation and Productivity Centre of Excellence
}

\author{
Sally Bridgwater \\ Numerical Algorithms Group \\ Oxford, UK \\ sally.bridgwater@nag.co.uk
}

\section{INDUSTRIAL EXTENDED ABSTRACT}

As machines get larger and scientific applications advance, it is more and more imperative to fully utilize high performance computing (HPC) capability. The complexity and changing landscape of parallel computers may lead to users being unable or unsure how to achieve optimal performance from their applications and fully utilize their HPC resources.

The Performance Optimisation and Productivity Centre of Excellence in Computing Applications (POP) has received funding from the European Commission as part of the Horizon 2020 programme to help alleviate these issues. It aims to uncover inefficiencies and their causes in existing parallel HPC applications that will lead to an improvement in the productivity and competitiveness of European organizations, in academia, government and industry. The POP project will drive efforts to highlight the need for and best practices in performance optimization through performance audits on codes along with training events to improve knowledge in this area. The aim is to help developers target their code development and refactoring in the most efficient direction and provide a return on investment from the savings due to the performance improvement.

The POP project combines the expertise and experience of Barcelona Supercomputing Center (BSC), High Performance Computing Center Stuttgart, Jülich Supercomputing Centre (JSC), Numerical Algorithms Group Ltd (NAG), RWTH Aachen and TERATEC. This combination provides longstanding and well respected resources in the academic and commercial realms. The POP members have come together to create a coherent and consistent methodology to give a clear, precise and useful overview of the performance of each HPC application.

The services of the POP project are free of charge to organizations with in the EU to analyze and advise on any parallel code in academic, government or industrial organizations of any domain.

\section{Methods Used}

Especially when aiming for very large scale HPC, efficiency of the application should be a major objective in program development. There is a broad need for detailed quantitative understanding of the actual cause of any inefficiencies and the complex interplay of effects as systems scale. This is a key need in development and refactoring of applications, especially as we work toward exascale machines.

There are two main performance analysis tools used during POP performance analysis. The Scalasca [1] environment has been developed at JSC and gives a highly scalable providing a call-path view. It can detect the root cause of many communication performance issues and provides a rich environment within which to investigate performance issues. The latest version of Scalasca is built upon Score-P [2] which is a community-developed parallel performance instrumentation and measurement system. It has an open format which allows the data to be used in conjunction with a range of tools. The second set of performance analysis tools have been developed by BSC are based around the Paraver [3] visualizer and Extrae trace collector. The data collection method used does not depend upon the programming or parallelization languages and so allows for a wide range of applications to be studied. It also scales well and so can easily be used to study production runs on HPC systems. The ability to use and create complex derived metrics and analyses gives the users a large degree of control and comparison can be made among different data sets.

The POP service provides an initial Application Performance Audit on each accepted code. This will give a clear overview of the performance of the application and highlight areas which could be improved upon with some insight into the issue from which the inefficiency arises. This is all written up into a report which may provide specific recommendations or highlight areas where deeper analysis is necessary before the issues can be fully pinpointed.

If further investigation is necessary, the POP team can undertake a Performance Plan which is a targeted investigation into difficult issues or a need for major refactoring. This refactoring may be demonstrated in a POP Proof-of-Concept.

In this poster the methodologies used will be highlighted using a completed Application Performance Audit. Areas of focus from the audit that will be discussed include the 
scalability, communication behavior, computational performance and load balance of the application.

\section{EXPECTED SIGNIFICANCE}

From its start date in October 2015, POP aims to analyze 150 codes in its first 2.5 years of operations and it will aim to provide $€ 3 \mathrm{M}$ worth of savings directly through the improved performance of the HPC codes analyzed. These savings will be due to the reduced running costs from faster applications and also to the increased competitiveness of companies being able to get products to market faster. This will also enable European organizations to perform larger simulations and more cutting edge research using their improved capabilities.

Overall in the first six months POP has completed Application Performance Audits of 11 parallel codes and is currently in the process of working on 22 more Application Performance Audits and three Proof-of-Concepts. This shows that POP has made a great start towards its goal of delivering improved performance to European HPC applications and is on target to analyze the desired number of applications. A wide range of applications have been investigated in areas including material science, computational fluid dynamics, finite elements and plasma turbulence.

These studies and improvements have shown that there is a need for such a service as POP offers and that there is a wide scope for gains in applications if developers have access to the right expertise, further information about the POP project can be found on the website, https://pop-coe.eu.

\section{Keywords-component; profiling; Paraver; Scalasca; Centre of Excellence; HPC; scalability; performance optimization}

\section{ACKNOWLEDGMENT}

The author would like to thank R. F. L. Evans and R. W. Chantrell, the developers of VAMPIRE, for allowing us to work with them on this project. Thanks are also due to N. Dingle, J. Boyle, W. Miah, E. Smyth and M. Dewar at NAG and to J. Giménez and C. Rosas from BSC.

\section{BIOGRAPHIES}

SALLY BRIDGWATER is an HPC Application Analyst at NAG Ltd, working as part of the Performance Optimisation and Productivity Centre of Excellence. She received an MSci in Mathematics and Physics from the University of Bristol in 2010 and her PhD in Computational Physics from the University of Warwick in 2014.

\section{REFERENCES}

[1] M. Geimer, F. Wolf, B. J. N. Wylie, E. Ábrahám, D. Becker and B. Mohr, "The Scalasca performance toolset architecture," Concurrency Computat.: Pract. Exper. 22:702-719, 2010.

[2] Andreas Knüpfer et al. "Score-P: A joint performance measurement runtime infrastructure for Periscope, Scalasca, TAU and Vampir," in Tools for High Performance Computing 2011, Springer Berlin Heidelberg, pp79-91.

[3] J. Labarta, J. Giménez, E. Martínez, P. González, H. Servat, G. Llort, X. Aguilar. "Scalability of Tracing and Visualization Tools 2005" http://www.bsc.es/paraver, last accessed April 2016. 